\title{
Multiplicity of solutions for singular semilinear elliptic equations in weighted Sobolev spaces
}

Gao Jia* and Long-jie Zhang

\section{"Correspondence:} gaojia89@163.com College of Science, University of Shanghai for Science and Technology, Shanghai, 200093, China

\begin{abstract}
A class of semilinear elliptic equations involving strong resonance or non-resonance is reconsidered here. The multiplicity of solutions is investigated by using the variational method, and the results complement earlier ones.

MSC: 35B34; 35J20; 35J61; 46E35

Keywords: multiplicity; variational method; semilinear elliptic equations; weighted Sobolev space
\end{abstract}

\section{Introduction}

In this paper we investigate the following singular semilinear problem:

$$
\mathcal{L} u-\lambda u \rho=-\rho f(x, u), \quad x \in \Omega,
$$

where

$$
\mathcal{L} u=-D_{i}\left(p_{i}^{1 / 2} p_{j}^{1 / 2} a_{i j} D_{j} u\right)+b u \rho,
$$

and $\Omega \subseteq \mathbb{R}^{N}$ is an open set (possibly unbounded).

In recent years, the existence and multiplicity of solutions for semilinear equations have been extensively studied by many authors (see [1-11]).

In 2001, Shapiro [12] studied a series of eigenvalue problems of singular quasilinear elliptic and parabolic equations in weighted Sobolev spaces, he obtained many existence results by using Galerkin method. Then Jia et al. continued to study the quasilinear elliptic equations (see [13-15]). However, their main results are only concerned with the existence of solutions without considering multiplicity of solutions.

Motivated by previous work, the aim of this paper is to establish the existence of infinitely many solutions of the elliptic equations in weighted Sobolev spaces, by using the Ekeland variation principle and the mountain-pass lemma.

We first assume that

$(L-1) \quad a_{i j}(x), b(x) \in C^{0}(\Omega) \cap L^{\infty}(\Omega), p_{i}(x), \rho(x) \in C^{0}(\Omega), i, j=1,2, \ldots, N$.

$(L-2) p_{i}(x)>0, \rho(x)>0, \forall x \in \Omega$, and there exists $\varepsilon_{0}>0$ such that $b(x) \geq \varepsilon_{0}, \forall x \in \Omega$. 
$(L-3) \int_{\Omega} p_{i}<\infty, \int_{\Omega} \rho<\infty$.

$(L-4) a_{i j}(x)=a_{j i}(x), i, j=1,2, \ldots, N$.

$(L-5) a_{i j} \xi_{i} \xi_{j} \geq a_{0}|\xi|^{2}, a_{0}$ is a positive constant.

Let $\Gamma \subset \partial \Omega$ designate a fixed closed set ( $\Gamma$ may be empty).

We introduce the pre-Hilbert space:

$$
C_{p, \rho}^{1}(\Omega, \Gamma)=\left\{u \in C^{0}(\bar{\Omega}) \cap C^{2}(\Omega) \mid u(x)=0, \forall x \in \Gamma ; \int_{\Omega}\left(\left|D_{i} u\right|^{2} p_{i}+u^{2} \rho\right)<\infty\right\},
$$

with the inner product

$$
\langle u, v\rangle_{p, \rho}=\int_{\Omega}\left(D_{i} u D_{i} v p_{i}+u v \rho\right) .
$$

Let $H_{p, \rho}^{1}(\Omega, \Gamma)$ denote the Hilbert space completed by the norm $\|u\|_{p, \rho}=\langle u, u\rangle_{p, \rho}^{1 / 2}, L_{\rho}^{2}(\Omega)$ denote the Hilbert space with the inner product $\langle u, v\rangle_{\rho}=\int_{\Omega} u v \rho$.

Next, we define the two-form

$$
L(u, v)=\int_{\Omega}\left(p_{i}^{1 / 2} p_{j}^{1 / 2} a_{i j} D_{i} u D_{j} v+b \rho u v\right) .
$$

Definition 1.1 We say $(\Omega, \Gamma)$ is a $V_{L}$-region if the following two facts obtain:

$\left(V_{L}-1\right)$ There exists a complete orthonormal system $\left\{\varphi_{n}\right\}_{n=1}^{\infty}$ in $L_{\rho}^{2}(\Omega)$, and $m\left(\left\{x \in \Omega \mid \varphi_{i}=\right.\right.$ $0\})=0$. Also $\varphi_{n} \in H_{p, \rho}^{1}(\Omega, \Gamma) \cap C^{2}(\Omega)$.

$\left(V_{L}-2\right)$ There exists a sequence of eigenvalues $\left\{\lambda_{n}\right\}_{n=1}^{\infty}$ with $0<\lambda_{1} \leq \lambda_{2} \leq \lambda_{3} \leq \cdots \leq \lambda_{n} \rightarrow$ $\infty$ such that $L\left(\varphi_{n}, v\right)=\lambda_{n}\left\langle\varphi_{n}, v\right\rangle_{\rho}, \forall v \in H_{p, \rho}^{1}(\Omega, \Gamma)$. Also $\varphi_{1}>0$ in $\Omega$.

\section{Remark 1.1}

$$
\begin{aligned}
& \lambda_{k+1}=\inf \left\{L(u, u) \mid u \in V_{k}^{\perp},\|u\|_{L_{\rho}^{2}}=1\right\}, \\
& L(u, u) \leq \lambda_{k} \int_{\Omega} u^{2} \rho, \quad u \in V_{k}, k \geq 1, \\
& L(u, u) \geq \lambda_{k+1} \int_{\Omega} u^{2} \rho, \quad u \in V_{k}^{\perp}, k \geq 0,
\end{aligned}
$$

where $V_{k}=\operatorname{span}\left\{\varphi_{1}, \varphi_{2}, \ldots, \varphi_{k}\right\}$.

Definition 1.2 We say $(\Omega, \Gamma)$ is a simple $V_{L}$-region if $(\Omega, \Gamma)$ is a $V_{L}$-region and the following four conditions are satisfied:

$\left(S V_{L}-1\right) \quad \Omega=\Omega_{1} \times \Omega_{2} \times \cdots \times \Omega_{N}$.

$\left(S V_{L}-2\right)$ Associated with each $\Omega_{i}$ there are positive functions $p_{i}^{*}$ and $\rho_{i}^{*}$ in $C^{0}(\Omega)$ satisfying $\int_{\Omega_{i}}\left(p_{i}^{*}+\rho_{i}^{*}\right)<\infty$, for $i=1,2, \ldots, N$.

$\left(S V_{L}-3\right) \rho(x)=\rho_{1}^{*}\left(x_{1}\right) \cdots \rho_{N}^{*}\left(x_{N}\right)$ and

$$
p_{i}(x)=\rho_{1}^{*}\left(x_{1}\right) \cdots \rho_{i-1}^{*}\left(x_{i-1}\right) p_{i}^{*}\left(x_{i}\right) \rho_{i+1}^{*}\left(x_{i+1}\right) \cdots \rho_{N}^{*}\left(x_{N}\right)
$$

for $i=1, \ldots, N$. 
$\left(S V_{L}-4\right)$ For each $\Omega_{i}(i=1, \ldots, N), \exists h_{i} \in C^{0}\left(\Omega_{i}\right) \cap L_{\rho_{i}^{*}}^{\theta}\left(\Omega_{i}\right)$, for $2<\theta<\infty$, with the property that

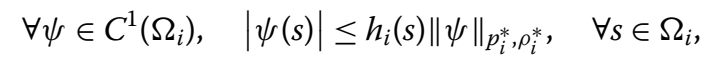

where $h_{i}$ is in $L_{\rho_{i}^{*}}^{\theta}\left(\Omega_{i}\right)$ for $2<\theta$, and also, to be quite explicit,

$$
\|\psi\|_{p_{i}^{*}, \rho_{i}^{*}}^{2}=\int_{\Omega_{i}}\left(p_{i}^{*}(s)\left|\psi^{\prime}(s)\right|^{2}+\rho_{i}^{*} \psi^{2}(s)\right)
$$

In fact, there are many examples which use special functions to illustrate the $V_{L}$-region or the simple $V_{L}$-region (see [12]).

The weak solutions of (1.1) correspond to the critical points of the functional $I_{\lambda}(u)$ : $H_{p, \rho}^{1}(\omega, \Gamma) \rightarrow \mathbb{R}$ defined by

$$
I_{\lambda}(u)=\frac{1}{2} L(u, u)-\frac{\lambda}{2} \int_{\Omega} u^{2} \rho+\int_{\Omega} F(x, u) \rho, \quad u \in H_{p, \rho}^{1}(\Omega, \Gamma),
$$

where $F(x, s) \in C^{1}(\bar{\Omega} \times \mathbb{R}, \mathbb{R})$ and $F(x, s)=\int_{0}^{s} f(x, t) d t$.

We define

$$
\begin{cases}T^{+}=\liminf _{s \rightarrow+\infty} F(x, s), & S^{+}=\limsup _{s \rightarrow+\infty} F(x, s), \\ T^{-}=\liminf _{s \rightarrow-\infty} F(x, s), & S^{-}=\lim \sup _{s \rightarrow-\infty} F(x, s) .\end{cases}
$$

Here, the above functions belong to $L_{\rho}^{1}(\Omega)$ and the limits are taken a.e. and uniformly in $x \in \Omega$.

As for $f(x, s)$ and $F(x, s)$, we make the assumptions as follows.

$(F-1)$ There exists a function $k(x)>0, k(x) \in L_{\rho}^{1}(\Omega)$, such that

$$
\lim _{|s| \rightarrow+\infty} f(x, s)=0, \quad|F(x, s)| \leq k(x), \quad \text { a.e. } x \in \Omega, \forall s \in \mathbb{R}
$$

$(F-2)$ There exists a constant $b_{1} \geq 0$ such that

$$
F(x, s) \geq \frac{1}{2}\left(\lambda_{1}-\lambda_{2}\right) s^{2}+b_{1}\left(\int_{\Omega} \rho\right)^{-1}
$$

$(F-3) \quad \int_{\Omega} S^{+} \rho \leq 0$ and $\int_{\Omega} S^{-} \rho \leq 0$.

$(F-4)$ There exists $t_{0} \in \mathbb{R}$ such that for the first eigenfunction $\varphi_{1}$

$$
\int_{\Omega} F\left(x, t_{0} \varphi_{1}\right) \rho<\min \left\{\int_{\Omega} T^{+} \rho, \int_{\Omega} T^{-} \rho\right\}
$$

$(F-5)$ There exists $t_{1}^{ \pm} \in \mathbb{R}$ such that for the first eigenfunction $\varphi_{1}$

$$
\int_{\Omega} F\left(x, t_{1}^{ \pm} \varphi_{1}\right) \rho<\min \left\{\int_{\Omega} T^{+} \rho, \int_{\Omega} T^{-} \rho\right\} .
$$


$(F-6)$ There exist $\alpha \in(0,1)$ and $\delta>0$ such that

$$
F(x, s) \geq \frac{1-\alpha}{2} \lambda_{1} s^{2}, \quad \forall x \in \Omega \text { and }|s|<\delta .
$$

$\left(F^{\prime}-1\right)$ Let $\eta>2, f(x,-s)=-f(x, s)$ a.e. $x \in \mathbb{R}^{N}, \forall s \in \mathbb{R}$ and

$$
0>\frac{1}{\eta} F(x, s) \geq f(x, s) s, \quad \text { a.e. } x \in \mathbb{R}^{N}, \forall s \in \mathbb{R} \text {. }
$$

$\left(F^{\prime}-2\right)$ There exists $1<\theta<\frac{N+1}{N-1}$ such that

$$
|f(x, s)| \leq C|s|^{\theta}, \quad \text { a.e. } x \in \mathbb{R}^{N}, \forall s \in \mathbb{R}
$$

for some positive constant $C$.

$\left(F_{k}-1\right) \int_{\Omega \cap\{\omega>0\}} S^{+} \rho+\int_{\Omega \cap\{\omega<0\}} S^{-} \rho \leq 0$, for every $\omega \in V\left(\lambda_{k}\right)$, where $V\left(\lambda_{k}\right)$ denotes the eigenspace associated with the eigenvalue $\lambda_{k}$.

$\left(F_{k}-2\right)$ There exist $\gamma \in\left(\frac{\lambda_{k}}{2}-\frac{\lambda_{1}}{2}, \frac{\lambda_{k}}{2}\right)$ and $\delta>0$ such that

$$
F(x, s) \geq \gamma s^{2}, \quad \forall x \in \Omega \text { and }|s|<\delta
$$

Remark 1.2 We must find the function $F(x, s)$ satisfying $(F-1)-(F-6)$. Let $\Omega=(0, \pi)$, $\lambda_{1}$, and $\lambda_{2}$ be the first eigenvalue and second eigenvalue of Laplacian eigenvalue problem of homogeneous boundary condition on $\Omega$, and the first eigenfunction be $\phi_{1}=\sin x$. Let $F(x, s)=\frac{1}{4}\left(\lambda_{2}-\lambda_{1}\right)\left(2 e^{-s^{2}}-1\right)$. For $b_{1} \equiv 0, \rho \equiv 1$, by simple calculation, if $t_{0}$ is large enough, we find that $F(x, s)$ satisfies $(F-1)-(F-6)$.

On the other hand, if we take $f(x, s)=-|s|^{k-1} s$, where $1<k<\frac{N+1}{N-1}$, then $f(x, s)$ satisfies $\left(F^{\prime}-1\right)$ and $\left(F^{\prime}-2\right)$.

The main results of our paper are given by the following theorems.

Theorem 1.1 Let $\Omega \subseteq \mathbb{R}^{N}$ be a domain satisfying the requirements of being a $V_{L}$-region. Let $\lambda=\lambda_{1}$ in (1.1). Assume that $F(x, s) \in C^{1}(\bar{\Omega} \times \mathbb{R}, \mathbb{R})$. Furthermore, if the conditions $(F-1)$, $(F-3)$, and $(F-4)$ are satisfied, then the problem (1.1) has at least one nontrivial solution in $H_{p, \rho}^{1}(\Omega, \Gamma)$.

Theorem 1.2 Let $\Omega \subseteq \mathbb{R}^{N}$ be a domain satisfying $V_{L}$-region. And $\lambda=\lambda_{1}$ in (1.1). Assume that $F(x, s) \in C^{1}(\bar{\Omega} \times \mathbb{R}, \mathbb{R})$. Furthermore, if the conditions $(F-1)-(F-3)$ and $(F-5)$ are satisfied, then the problem (1.1) has at least two nontrivial solutions in $H_{p, \rho}^{1}(\Omega, \Gamma)$.

Theorem 1.3 Let $\Omega \subseteq \mathbb{R}^{N}$ be a domain satisfying simple $V_{L}$-region, $\left(a_{i j}\right)=E$, and $\lambda=\lambda_{1}$. Assume that $F(x, s) \in C^{1}(\bar{\Omega} \times \mathbb{R}, \mathbb{R})$. Furthermore, if the conditions $(F-1),(F-3),(F-4)$, and $(F-6)$ are satisfied, then the problem (1.1) has at least two nontrivial solutions in $H_{p, \rho}^{1}(\Omega, \Gamma)$.

Theorem 1.4 Let $\Omega \subseteq \mathbb{R}^{N}$ be a domain satisfying simple $V_{L}$-region, $\left(a_{i j}\right)=E$, and $\lambda=\lambda_{1}$. Assume that $F(x, s) \in C^{1}(\bar{\Omega} \times \mathbb{R}, \mathbb{R})$. Furthermore, if the conditions $(F-1)-(F-3),(F-5)$, and $(F-6)$ are satisfied, then the problem (1.1) has at least three nontrivial solutions in $H_{p, \rho}^{1}(\Omega, \Gamma)$. 
Theorem 1.5 Let $\Omega \subseteq \mathbb{R}^{N}$ be a domain satisfying simple $V_{L}$-region, $\lambda<\lambda_{1}$ and $\left(a_{i j}\right)=E$. As sume that $F(x, s) \in C^{1}(\bar{\Omega} \times \mathbb{R}, \mathbb{R})$. Furthermore, suppose the conditions $\left(F^{\prime}-1\right)$ and $\left(F^{\prime}-2\right)$. Then the problem (1.1) has infinitely many solutions in $H_{p, \rho}^{1}(\Omega, \Gamma)$.

Theorem 1.6 Let $\Omega \subseteq \mathbb{R}^{N}$ be a domain satisfying simple $V_{L}$-region, $\left(a_{i j}\right)=E$. $\lambda=\lambda_{k}$ $(k \geq 2)$. Assume that $F(x, s) \in C^{1}(\bar{\Omega} \times \mathbb{R}, \mathbb{R})$. Furthermore, suppose the conditions $(F-1)$, $\left(F_{k}-1\right)$, and $\left(F_{k}-2\right)$. Then the problem (1.1) has two nontrivial solutions in $H_{p, \rho}^{1}(\Omega, \Gamma)$.

The paper is organized as follows. In Section 2, we provide and establish some lemmas which are necessary in the proof of our main theorems. In Section 3, we will prove Theorem 1.1-Theorem 1.5. In the last section, we establish Lemma 4.1 and Lemma 4.2, and we give the proof of Theorem 1.6.

\section{Preliminary results}

In this section, we prove some lemmas which will be used in the proof of our main theorems. For simplicity, we denote $I_{\lambda}(\cdot)$ by $I(\cdot)$ in the following.

Lemma 2.1 Let $L$ be defined as (1.2). Then

$$
c_{2}\|u\|_{p, \rho}^{2} \geq L(u, u) \geq c_{1}\|u\|_{p, \rho}^{2}, \quad \forall u \in H_{p, \rho}^{1}(\Omega, \Gamma)
$$

for some positive constants $c_{1}, c_{2}$.

To get Lemma 2.7 and Lemma 2.8, we first introduce a corollary of the Ekeland variation principle (see [16], Theorem 2.4).

Lemma 2.2 Let $X$ be a Banach space. Assume that $E \in C^{1}(X, \mathbb{R})$ is bounded from below, which satisfies the $(P S)_{c}$ condition. Then $c=\inf _{x \in X} E(x)$ is a critical value.

Lemma 2.3 Assume that $L$ is given by (1.2), and the assumptions $(L-1)-(L-5)$ hold, and that $(\Omega, \Gamma)$ is a $V_{L}$-region. Then $H_{p, \rho}^{1}(\Omega, \Gamma)$ is compactly embedding in $L_{\rho}^{2}$. Moreover, if $(\Omega, \Gamma)$ is a simple $V_{L}$-region and $\left(a_{i j}\right)=E$, then for $N \geq 2, H_{p, \rho}^{1}(\Omega, \Gamma)$ is compactly embedded in $L_{\rho}^{\theta}, 2<\theta<\frac{2 N}{N-1}$.

Proof See Lemma 2 and Theorem 9 in [12].

To establish the multiplicity of solutions for problem (1.1), we need to apply the following fundamental theorem (see [17], Theorem 9.12).

Lemma 2.4 Let $X$ be an infinite-dimensional Banach space and let $E: X \rightarrow \mathbb{R}$ be continuous, even and satisfying $(P S)_{c}$ for every $c \in \mathbb{R}$. Assume, also, that:

(1) There exist $\rho>0, \alpha>E(0)$, and a subspace $V \subset X$ of finite codimension such that

$$
\forall u \in V: \quad\|u\|=\rho \quad \Rightarrow \quad E(u) \geq \alpha .
$$

(2) For every finite-dimensional subspace $W_{n} \subset X, \operatorname{dim}\left(W_{n}\right)=n$, there exists $R_{n}>0$ such that

$$
\forall u \in W_{n}: \quad\|u\|=R_{n} \quad \Rightarrow \quad E(u) \leq E(0) .
$$

Then there exists a sequence $\left\{h_{n}\right\}$ of critical values of $E$ with $h_{n} \rightarrow+\infty$. 
Next, we describe some results under the geometry for functional $I$.

Lemma 2.5 Under hypotheses $(F-1)$ and $(F-2),(\Omega, \Gamma)$ is a $V_{L}$-region, the functional $I$ has the following saddle geometry for $\lambda=\lambda_{1}$ :

(1) $I(u) \rightarrow \infty$ if $\|u\|_{p, \rho} \rightarrow \infty$ with $u \in V_{1}^{\perp}$.

(2) There is $\alpha \in \mathbb{R}$ such that $I(u) \leq \alpha, \forall u \in V_{1}$.

(3) $I(u) \geq b_{1}, \forall u \in V_{1}^{\perp}$, where $b_{1}$ is given by $(F-2)$.

Proof (1) By Remark 1.1, we have

$$
\begin{aligned}
I(u) & \geq \frac{1}{2}\left(1-\frac{\lambda_{1}}{\lambda_{2}}\right) L(u, u)+\int_{\Omega} F(x, u) \rho \\
& \geq \frac{c_{1}}{2}\left(1-\frac{\lambda_{1}}{\lambda_{2}}\right)\|u\|_{p, \rho}^{2}+\int_{\Omega} F(x, u) \rho, \quad u \in V_{1}^{\perp} .
\end{aligned}
$$

Using $(F-1)$, we have $I(u) \rightarrow \infty$, as $\|u\|_{p, \rho} \rightarrow \infty$.

(2) By a simple calculation, we get

$$
I(u)=\int_{\Omega} F(x, u), \quad u \in V_{1} .
$$

By using $(F-1)$ we have

$$
I(u)=\int_{\Omega} F(x, u) \leq \int_{\Omega} k(x) \rho .
$$

So we choose $\alpha=\int_{\Omega} k(x) \rho$.

(3) By $(F-2)$ and Remark 1.1, we get

$$
I(u) \geq \frac{1}{2} L(u, u)-\frac{\lambda_{2}}{2} \int_{\Omega} u^{2} \rho+b_{1} \geq b_{1}, \quad \forall u \in V_{1}^{\perp},
$$

the proof of this lemma is completed.

Next, we will prove the Palais-Smale properties for the functional $I$. We recall that $I$ : $E \rightarrow \mathbb{R}$ satisfies the Palais-Smale conditions at the level $c \in \mathbb{R}\left((P S)_{c}\right.$ in short). For any sequence $\left\{h_{n}\right\} \subset E$ such that

$$
I\left(h_{n}\right) \rightarrow c, \quad I^{\prime}\left(h_{n}\right) \rightarrow 0,
$$

as $n \rightarrow \infty$, the sequence $\left\{h_{n}\right\}$ possesses a convergent subsequence in $E$. Moreover, we say that $I$ satisfies $(P S)$ conditions when we have $(P S)_{c}$ for all $c \in \mathbb{R}$ (see [16]).

Lemma 2.6 Assume the condition $(F-1)$ holds, $\lambda=\lambda_{1}$, and $(\Omega, \Gamma)$ is a $V_{L}$-region. Then the functional I has the $(P S)_{c}$ conditions whenever $c<\min \left\{\int_{\Omega} T^{+} \rho, \int_{\Omega} T^{-} \rho\right\}$ or $c>\max \left\{\int_{\Omega} S^{+} \rho, \int_{\Omega} S^{-} \rho\right\}$.

Proof We only prove the lemma for $c<\min \left\{\int_{\Omega} T^{+} \rho, \int_{\Omega} T^{-} \rho\right\}$. For the case $c>\max \left\{\int_{\Omega} S^{+} \rho\right.$, $\left.\int_{\Omega} S^{-} \rho\right\}$, we can use similar methods. 
1. The boundedness of $(P S)$ sequence.

If this is not the case, there exists a $(P S)_{c}$ unbounded sequence $\left\{u_{n}\right\} \in H_{p, \rho}^{1}(\Omega, \Gamma)$ such that $c<\min \left\{\int_{\Omega} T^{+} \rho, \int_{\Omega} T^{-} \rho\right\}$. Without loss of generality, we may assume that, as $n \rightarrow \infty$, the following expressions hold:

$$
\left\|u_{n}\right\|_{p, \rho} \rightarrow \infty, \quad I\left(u_{n}\right) \rightarrow c, \quad I^{\prime}\left(u_{n}\right) \rightarrow 0
$$

Define $\bar{u}_{n}=\frac{u_{n}}{\left\|u_{n}\right\|_{p, \rho}}$. Hence by Lemma 2.3, there is an $\bar{u} \in H_{p, \rho}^{1}(\Omega, \Gamma)$ with the following properties:

$$
\begin{aligned}
& \bar{u}_{n} \rightarrow \bar{u} \text { in } H_{p, \rho}^{1}(\Omega, \Gamma), \\
& \bar{u}_{n} \rightarrow \bar{u} \text { in } L_{\rho}^{2}(\Omega), \\
& \bar{u}_{n} \rightarrow \bar{u} \text { a.e. in } \Omega .
\end{aligned}
$$

For any $\Phi \in H_{p, \rho}^{1}(\Omega, \Gamma)$, it is obvious that $\frac{I^{\prime}\left(u_{n}\right) \Phi}{\left\|u_{n}\right\|_{p, \rho}} \rightarrow 0$. By the convergence of $\left\{\bar{u}_{n}\right\}$ and $(F-1)$, we have

$$
L(\bar{u}, \Phi)=\lambda_{1} \int_{\Omega} \bar{u} \Phi \rho .
$$

According to the definition of $\lambda_{1}$, we obtain $\bar{u}= \pm \varphi_{1}$. So, we suppose initially that $\bar{u}=\varphi_{1}$. Because $\varphi_{1}>0$, it is obvious that $u_{n} \rightarrow+\infty$, a.e. $x \in \Omega$ as $n \rightarrow \infty$.

Hence, taking $u_{n}=t_{n} \varphi_{1}+\omega_{n},\left\{t_{n}\right\} \subset \mathbb{R},\left\{\omega_{n}\right\} \subset V_{1}^{\perp}$, by Remark 1.1, we have

$$
I\left(u_{n}\right) \geq \frac{1}{2}\left(1-\frac{\lambda_{1}}{\lambda_{2}}\right) L\left(\omega_{n}, \omega_{n}\right)+\int_{\Omega} F\left(x, u_{n}\right) \rho .
$$

Since $I\left(u_{n}\right) \rightarrow c$, it can easily be concluded that the sequence $\left\{\omega_{n}\right\}$ is bounded. Because of $\left\|u_{n}\right\| \rightarrow \infty$, on a subsequence $\left|t_{n}\right| \rightarrow \infty$, without loss of generality, we assume $t_{n} \rightarrow+\infty$.

Now, by the Hölder inequality and $(F-1)$, we have

$$
\left|\int_{\Omega} f\left(x, u_{n}\right) \omega_{n} \rho\right| \leq C\left(\int_{\Omega}\left|f\left(x, u_{n}\right)\right|^{2} \rho\right)^{\frac{1}{2}} .
$$

Thus, by applying the dominated convergence theorem, we conclude that

$$
\lim _{n \rightarrow \infty} \int_{\Omega} f\left(x, u_{n}\right) \omega_{n} \rho=0
$$

On the other hand,

$$
I^{\prime}\left(u_{n}\right) \omega_{n}=L\left(\omega_{n}, \omega_{n}\right)-\lambda_{1} \int_{\Omega} \omega_{n}^{2} \rho+\int_{\Omega} f\left(x, u_{n}\right) \omega_{n} \rho,
$$

by (2.2) and Remark 1.1, we obtain

$$
\left(1-\frac{\lambda_{1}}{\lambda_{2}}\right) L\left(\omega_{n}, \omega_{n}\right) \leq\left|I^{\prime}\left(u_{n}\right) \omega_{n}\right|+\left|\int_{\Omega} f\left(x, u_{n}\right) \omega_{n} \rho\right| \rightarrow 0,
$$

as $n \rightarrow \infty$. Therefore, due to Remark 1.1, we have

$$
L\left(\omega_{n}, \omega_{n}\right)-\lambda_{1} \int_{\Omega} \omega_{n}^{2} \rho \rightarrow 0, \quad \text { as } n \rightarrow \infty .
$$


Consequently, by virtue of Fatou's lemma and assumption $(F-1)$, we get

$$
\begin{aligned}
c & =\lim _{n \rightarrow \infty}\left(L\left(\omega_{n}, \omega_{n}\right)-\lambda_{1} \int_{\Omega} \omega_{n}^{2} \rho+\int_{\Omega} F\left(x, u_{n}\right) \rho\right) \\
& \geq \int_{\Omega} T^{+} \rho,
\end{aligned}
$$

which contradicts with the condition $c<\min \left\{\int_{\Omega} T^{+} \rho, \int_{\Omega} T^{-} \rho\right\}$. Hence, the $(P S)_{c}$ sequence of the functional $I$ is bounded.

2. Various convergences of $\left\{u_{n}\right\}$.

Since $\left\{u_{n}\right\}$ is a bounded sequence, there is an $u \in H_{p, \rho}^{1}(\Omega, \Gamma)$ with the following properties:

$$
\begin{aligned}
& u_{n} \rightarrow u \text { in } H_{p, \rho}^{1}(\Omega, \Gamma), \\
& u_{n} \rightarrow u \text { in } L_{\rho}^{2}(\Omega), \\
& u_{n} \rightarrow u \text { a.e. in } \Omega .
\end{aligned}
$$

3. $\left\{u_{n}\right\}$ converges to $u$ in $H_{p, \rho}^{1}(\Omega, \Gamma)$.

From the definition of $(P S)_{c}$ sequence, we have, as $n \rightarrow \infty$,

$$
\begin{aligned}
& I^{\prime}\left(u_{n}\right) u=L\left(u_{n}, u\right)-\lambda_{1} \int_{\Omega} u_{n} u \rho+\int_{\Omega} f\left(x, u_{n}\right) u \rho \rightarrow 0, \\
& I^{\prime}\left(u_{n}\right) u_{n}=L\left(u_{n}, u_{n}\right)-\lambda_{1} \int_{\Omega} u_{n}^{2} \rho+\int_{\Omega} f\left(x, u_{n}\right) u_{n} \rho \rightarrow 0 .
\end{aligned}
$$

By Fatou's lemma and the above convergence of $\left\{u_{n}\right\}$, it is easy to show that

$$
\begin{aligned}
& \int_{\Omega} u_{n} u \rho \rightarrow \int_{\Omega} u^{2} \rho, \\
& \int_{\Omega} f\left(x, u_{n}\right) u \rho \rightarrow \int_{\Omega} f(x, u) u \rho, \\
& \int_{\Omega} u_{n}^{2} \rho \rightarrow \int_{\Omega} u^{2} \rho, \\
& \int_{\Omega} f\left(x, u_{n}\right) u_{n} \rho \rightarrow \int_{\Omega} f(x, u) u \rho,
\end{aligned}
$$

as $n \rightarrow \infty$. Hence, we get

$$
\begin{aligned}
& L\left(u_{n}, u\right) \rightarrow \lambda_{1} \int_{\Omega} u^{2} \rho-\int_{\Omega} f(x, u) u \rho, \quad \text { as } n \rightarrow \infty, \\
& L\left(u_{n}, u_{n}\right) \rightarrow \lambda_{1} \int_{\Omega} u^{2} \rho-\int_{\Omega} f(x, u) u \rho, \quad \text { as } n \rightarrow \infty .
\end{aligned}
$$

By the weak convergence, it follows that

$$
L\left(u_{n}, u\right) \rightarrow L(u, u), \quad \text { as } n \rightarrow \infty .
$$

By using (2.3), (2.4), (2.5), and a simple calculation, we obtain

$$
L\left(u_{n}-u, u_{n}-u\right) \rightarrow 0, \quad \text { as } n \rightarrow \infty .
$$

From Lemma 2.1, the proof is completed. 
Lemma 2.7 Suppose that $(F-1)$ and $(F-6)$ are satisfied, $\lambda=\lambda_{1}$ and $(\Omega, \Gamma)$ is a simple $V_{L}$-region. Then the origin is a local minimum for the functional $I$.

Proof From (1.4), it is easy to see that $I(0)=0$. By $(F-6)$, we can choose $q \in\left(2, \frac{2 N}{N-1}\right)$ and a constant $C>0$ such that

$$
F(x, s) \geq \frac{1-\alpha}{2} \lambda_{1} s^{2}-C|s|^{q}, \quad \forall(x, s) \in \Omega \times \mathbb{R} .
$$

Consequently, by Remark 1.1 and Lemma 2.3, we have

$$
I(u) \geq \frac{1}{2}(1-\alpha) c_{1}\|u\|_{p, \rho}^{2}-C\|u\|_{p, \rho}^{q} \geq \frac{1}{4}(1-\alpha) c_{1}\|u\|_{p, \rho}^{2}, \quad\|u\|_{p, \rho}<r,
$$

where $r$ is small enough and $0<r<t_{0}, t_{0}$ is provided by $(F-4)$. Therefore the proof is completed.

To complete the mountain-pass geometry, we also need the following result.

Lemma 2.8 Let the hypotheses $(F-1),(F-3),(F-4)$, and $(F-6)$ hold, $\lambda=\lambda_{1}$ and let $(\Omega, \Gamma)$ be a $V_{L}$-region. Then there exists $u_{0} \in H_{p, \rho}^{1}(\Omega, \Gamma)$ such that $I\left(u_{0}\right)<0$ and $\left\|u_{0}\right\|_{p, \rho}>r$, where $r$ is given by Lemma 2.7 .

Proof By $(F-1)$ and $(F-4)$, we take $u_{0}=t_{0} \varphi_{1}$ where $t_{0}$ is provided by $(F-4)$. Thus, we obtain

$$
I\left(t_{0} \varphi_{1}\right)<\min \left\{\int_{\Omega} T^{+} \rho, \int_{\Omega} T^{-} \rho\right\}<\max \left\{\int_{\Omega} S^{+} \rho, \int_{\Omega} S^{-} \rho\right\} \leq 0
$$

and $\left\|t_{0} \Phi_{1}\right\|=t_{0}$. By Lemma 2.7, we have $0<r<t_{0}$, then the conclusion follows.

Lemma 2.9 If hypotheses $(F-1),(F-3)$, and $(F-4)$ are satisfied, $\lambda=\lambda_{1}$ and $(\Omega, \Gamma)$ is a $V_{L^{-}}$ region. Then problem (1.1) has at least one nontrivial solution $u_{0} \in H_{p, \rho}^{1}(\Omega, \Gamma)$. Moreover, $u_{0}$ has negative energy, i.e. $I\left(u_{0}\right)<0$.

Proof By $(F-1)$ and Remark 1.1, we have

$$
I(u) \geq \int_{\Omega} F(x, u) \rho \geq-\int_{\Omega} k(x) \rho .
$$

Therefore, the functional $I$ is bounded below. In this case, we would like to mention that the functional $I$ satisfies the $(P S)_{c}$ conditions with $c=\inf \left\{I(h): h \in H_{p, \rho}^{1}(\Omega, \Gamma)\right\}$. To see this, by Lemma 2.6 , we take $t_{0} \in \mathbb{R}$ provided by $(F-4)$, we obtain

$$
c \leq I\left(t_{0} \varphi_{1}\right)=\int_{\Omega} F\left(x, t_{0} \varphi_{1}\right)<\min \left\{\int_{\Omega} T^{+} \rho, \int_{\Omega} T^{-} \rho\right\} \leq 0 .
$$

Consequently, applying Lemma 2.2 we have one critical point $u_{0} \in H_{p, \rho}^{1}(\Omega, \Gamma)$ such that $I\left(u_{0}\right)=\inf \left\{I(h): h \in H_{p, \rho}^{1}(\Omega, \Gamma)\right\} \leq I\left(t_{0} \varphi_{1}\right)<0$. The proof of this lemma is completed.

To prove Theorem 1.3, we establish the following lemma. 
Lemma 2.10 Assume that the conditions $(F-1),(F-2),(F-3)$, and $(F-5)$ are satisfied, $\lambda=\lambda_{1}$ and $(\Omega, \Gamma)$ is a $V_{L}$-region. Then the problem (1.1) has at least two nontrivial solutions with negative energy.

Proof Define

$$
M^{+}=\left\{t \varphi_{1}+\omega, t \geq 0, \omega \in V_{1}^{\perp}\right\}, \quad M^{-}=\left\{t \varphi_{1}+\omega, t \leq 0, \omega \in V_{1}^{\perp}\right\} .
$$

We have $\partial M^{+}=\partial M^{-}=V_{1}^{\perp}$. Hence, we minimizer the functional $I$ restrict to $M^{+}$and $M^{-}$.

Firstly, we consider the functionals $I^{ \pm}=\left.I\right|_{M^{ \pm}}$. By the assumption $(F-4)$, we have

$$
\begin{aligned}
c^{ \pm} & =\inf \left\{I^{ \pm}, h \in E\right\} \leq I^{ \pm}\left(t_{1}^{ \pm} \varphi_{1}\right)=\int_{\Omega} F\left(x, t_{1}^{ \pm} \varphi_{1}\right) \rho \\
& <\min \left\{\int_{\Omega} T^{+} \rho, \int_{\Omega} T^{-} \rho\right\} \leq 0 .
\end{aligned}
$$

By Lemma 2.6, $I^{ \pm}$satisfy the $(P S)_{c}$ conditions whenever $c<\min \left\{\int_{\Omega} T^{+} \rho, \int_{\Omega} T^{-} \rho\right\}$. Therefore, we find that $I^{ \pm}$satisfy the $(P S)_{c}$ conditions with $c^{ \pm}=\inf \left\{I^{ \pm}(h): h \in E\right\}$.

In this way, by using Lemma 2.2 for the functionals $I^{+}$and $I^{-}$, we obtain two critical points denoted by $u_{0}^{+}$and $u_{0}^{-}$, respectively. Thus, we have $c^{+}=I^{+}\left(u_{0}^{+}\right)=\inf _{h \in M^{+}}\{I(h)\}$ and $c^{-}=I^{-}\left(u_{0}^{-}\right)=\inf _{h \in M^{-}}\{I(h)\}$.

Moreover, we affirm that $u_{0}^{+}$and $u_{0}^{-}$are nonzero critical points. Based on $(F-3)$ and $(F-5)$, we obtain

$$
I^{ \pm}\left(u_{0}^{ \pm}\right) \leq I^{ \pm}\left(t_{1}^{ \pm} \varphi_{1}\right)<\min \left\{\int_{\Omega} T^{+} \rho, \int_{\Omega} T^{-} \rho\right\} \leq 0,
$$

and $I$ is restricted to $V_{1}^{\perp}$ being nonnegative. More specifically, given $\omega \in V_{1}^{\perp}$ and (3) in Lemma 2.5, we have

$$
I(\omega) \geq b_{1} \geq 0 .
$$

Next, we prove that $u_{0}^{+}$and $u_{0}^{-}$are distinct. The proof of this affirmation is by contradiction. If $u_{0}^{+}=u_{0}^{-}$, then $u_{0}^{+}=u_{0}^{-} \in V_{1}^{\perp}$. By (2.6), we obtain $I\left(u_{0}^{+}\right)<0 \leq I\left(u_{0}^{+}\right)$. Therefore, we have a contradiction. Consequently, we get $u_{0}^{+} \neq u_{0}^{-}$. Thus the problem (1.1) has at least two nontrivial solutions. Moreover, these solutions have negative energy.

To prove Theorem 1.5, we need the following lemma. The proof is similar to that of Lemma 2.6.

Lemma 2.11 Assume that the conditions $\left(F^{\prime}-1\right)$ and $\left(F^{\prime}-2\right)$ hold, $\lambda<\lambda_{1},\left(a_{i j}\right)=E$ and $(\Omega, \Gamma)$ is a simple $V_{L}$-region. Then functional I satisfies the $(P S)_{c}$ conditions.

\section{Proofs of Theorem 1.1-Theorem 1.5}

In this section, we prove Theorems 1.1, 1.2, 1.3, 1.4, and 1.5.

Proof of Theorem 1.1 By Lemma 2.9, we get a solution $u_{0}$ which satisfies $I\left(u_{0}\right)<0$. It follows that the problem (1.1) has at least one nontrivial solution. The proof is completed. 
Proof of Theorem 1.2 Using Lemma 2.10, we obtain two distinct critical points $u_{0}^{+}$and $u_{0}^{-}$ such that $I\left(u_{0}^{+}\right)<0$ and $I\left(u_{0}^{-}\right)<0$. Therefore, the problem (1.1) has at least two nontrivial solutions. The proof is completed.

Proof of Theorem 1.3 From Lemma 2.7 and Lemma 2.8, we know that the functional $I$ satisfies the geometric conditions of the mountain-pass theorem. Moreover, the functional $I$ satisfies the $(P S)_{c}$ conditions for all $c \geq 0$. Thus, we have a solution $u_{2} \in H_{p, \rho}^{1}(\Omega, \Gamma)$ given by the mountain-pass theorem. Obviously, the solution $u_{2}$ satisfies $I\left(u_{2}\right)>0$.

On the other hand, by Lemma 2.9 , we get another solution $u_{0}$ satisfying $I\left(u_{0}\right)<0$. It follows that the problem (1.1) has at least two nontrivial solutions.

Proof of Theorem 1.4 The conditions $(F-1),(F-3),(F-5)$, and $(F-6)$ imply that Lemma 2.7 and Lemma 2.8 hold. Thus, we have one solution $u_{2}$ which satisfies $I\left(u_{2}\right)>0$.

On the other hand, using Lemma 2.10, we obtain two distinct critical points $u_{0}^{ \pm}$such that $I\left(u_{0}^{ \pm}\right)<0$. In other words, the problem (1.1) has at least three nontrivial solutions. The proof is completed.

Proof of Theorem 1.5 It is easy to see that functional $I$ is continuous and even. Moreover, by Lemma 2.11 , I satisfies $(P S)_{c}$ condition for every $c \in \mathbb{R}$. To prove Theorem 1.6 , we only need to test and verify the conditions (1) and (2) in Lemma 2.4.

1. By $\left(F^{\prime}-1\right)$ and Remark 1.1, we have

$$
I(u) \geq \frac{1}{2}\left(1-\frac{\lambda}{\lambda_{1}}\right) c_{1}\|u\|_{p, \rho}^{2}-C\|u\|_{p, \rho}^{\theta+1} .
$$

Hence there exist $r>0$ small enough and $\delta>0$ such that $I(u) \geq \delta$, for $\|u\|_{p, \rho}=r$. That is to say the condition (1) in Lemma 2.4 holds with $V=H_{p, \rho}^{1}(\Omega, \Gamma)$.

2. We verify the condition (2) in Lemma 2.4. Let $W$ be a finite-dimensional subspace of $H_{p, \rho}^{1}(\Omega, \Gamma)$. Let $u \in W$ such that $I(u) \geq 0$, i.e.

$$
\frac{1}{2} L(u, u)-\frac{\lambda}{2} \int_{\Omega} u^{2} \rho+\int_{\Omega} F(x, u) \rho \geq 0 .
$$

By $\left(F^{\prime}-1\right)$, there exist $m(x) \in L^{\infty}(\Omega)$ satisfying $m(x)>0$ a.e. $x \in \Omega$ and a positive constant $c$ such that

$$
F(x, s) \leq-m(x)|s|^{\eta}+c s^{2}, \quad \text { a.e. } x \in \Omega \text { and } \forall s \in \mathbb{R} \text {. }
$$

The inequality (3.1) implies

$$
c_{2}\|u\|_{p, \rho}^{2} \geq \int_{\Omega} m(x)|u|^{\eta} \rho-c \int_{\Omega} u^{2} \rho .
$$

Since $\left(\int_{\Omega} m(x)|u|^{\eta}\right)^{\frac{1}{\eta}}$ is a norm on $W$ and $W$ is finite-dimensional, then, by (3.2), there exists $c>0$ such that $\|u\|_{p, \rho}^{\eta} \leq c\|u\|_{p, \rho}^{2}$. Since $\eta>2$, we deduce that the set $\{u \in W, I(u) \geq 0\}$ is bounded in $H_{p, \rho}^{1}(\Omega, \Gamma)$ and the condition (2) in Lemma 2.4 holds.

\section{Proof of Theorem 1.6}

In this section, we consider the problem (1.1) in $\lambda=\lambda_{k}$. In order to prove Theorem 1.6, we first establish the following lemmas. 
Lemma 4.1 Assume that the condition $(F-1)$ holds, and that $(\Omega, \Gamma)$ is a $V_{L}$-region. Then the functional I satisfies the $(P S)_{c}$ conditions whenever $c<\inf _{\omega \in V\left(\lambda_{k}\right)}\left\{\int_{\Omega \cap\{\omega>0\}} T^{+} \rho+\right.$ $\left.\int_{\Omega \cap\{\omega<0\}} T^{-} \rho\right\}$ or $c>\sup _{\omega \in V\left(\lambda_{k}\right)}\left\{\int_{\Omega \cap\{\omega>0\}} S^{+} \rho+\int_{\Omega \cap\{\omega<0\}} S^{-} \rho\right\}$.

Proof We only prove the lemma for all $c<\inf _{\omega \in V\left(\lambda_{k}\right)}\left\{\int_{\Omega \cap\{\omega>0\}} T^{+} \rho+\int_{\Omega \cap\{\omega<0\}} T^{-} \rho\right\}$. For the case $c>\sup _{\omega \in V\left(\lambda_{k}\right)}\left\{\int_{\Omega \cap\{\omega>0\}} S^{+} \rho+\int_{\Omega \cap\{\omega<0\}} S^{-} \rho\right\}$, we can use similar methods.

The boundedness of $(P S)_{c}$ sequence.

Let us prove by contradiction. Suppose that there exists a $(P S)_{c}$ unbounded sequence $\left\{u_{n}\right\} \in H_{p, \rho}^{1}(\Omega, \Gamma)$ such that $c<\inf _{\omega \in V\left(\lambda_{k}\right)}\left\{\int_{\Omega \cap\{\omega>0\}} T^{+} \rho+\int_{\Omega \cap\{\omega<0\}} T^{-} \rho\right\}$. For ease of notation and without loss of generality, we assume that

$$
\left\|u_{n}\right\|_{p, \rho} \rightarrow \infty, \quad I\left(u_{n}\right) \rightarrow c, \quad I^{\prime}\left(u_{n}\right) \rightarrow 0, \quad n \rightarrow \infty .
$$

Define $\bar{u}_{n}=\frac{u_{n}}{\left\|u_{n}\right\|_{p, \rho}}$. Hence by Lemma 2.3, there is an $\bar{u} \in H_{p, \rho}^{1}(\Omega, \Gamma)$ with the following properties:

$$
\bar{u}_{n} \rightarrow \bar{u} \quad \text { in } H_{p, \rho}^{1}(\Omega, \Gamma), \quad \bar{u}_{n} \rightarrow \bar{u} \quad \text { in } L_{\rho}^{2}(\Omega), \quad \bar{u}_{n} \rightarrow \bar{u}, \quad \text { a.e. in } \Omega .
$$

For any $\Phi \in H_{p, \rho}^{1}(\Omega, \Gamma)$, we have $\frac{I^{\prime}\left(u_{n}\right) \Phi}{\left\|u_{n}\right\|_{p, \rho}} \rightarrow 0$. By the convergence of $\left\{\bar{u}_{n}\right\}$ and $(F-1)$, we have

$$
L(\bar{u}, \Phi)=\lambda_{k} \int_{\Omega} \bar{u} \Phi \rho
$$

By the definition of $\lambda_{k}$, we obtain $\bar{u} \in V\left(\lambda_{k}\right)$. It is obvious that $u_{n}(x) \rightarrow+\infty, x \in\{x \in \Omega \mid$ $\bar{u}(x)>0\}$ as $n \rightarrow \infty$, and $u_{n}(x) \rightarrow-\infty, x \in\{x \in \Omega \mid \bar{u}(x)<0\}$ as $n \rightarrow \infty$. By $\left(V_{L}-1\right)$, we have $m(\{x \in \Omega \mid \bar{u}(x)=0\})=0$.

Hence, we can take $u_{n}=\omega_{n}^{(1)}+\varphi_{n}+\omega_{n}^{(2)}$, where $\omega_{n}^{(1)} \in V_{1}, \varphi_{n} \in V\left(\lambda_{k}\right), \omega_{n}^{(2)} \in V_{2} . L_{\rho}^{2}=$ $V_{1} \oplus V\left(\lambda_{k}\right) \oplus V_{2}, V_{1}$ denotes the eigenspace associated to the eigenvalue $\lambda<\lambda_{k}$ and $V_{2}$ denotes the eigenspace associated to the eigenvalue $\lambda>\lambda_{k}$. Set

$$
\widetilde{u}_{n}=-\omega_{n}^{(1)}+\omega_{n}^{(2)} .
$$

By Remark 1.1, we have

$$
I^{\prime}\left(u_{n}\right) \widetilde{u}_{n} \geq \min \left\{\frac{\lambda_{k}}{\lambda_{k-1}}-1,1-\frac{\lambda_{k}}{\lambda_{k+1}}\right\} L\left(u_{n}-\varphi_{n}, u_{n}-\varphi_{n}\right)+\int_{\Omega} f\left(x, u_{n}\right) \tilde{u}_{n} \rho .
$$

Hence, by Lemma 2.1, we get

$$
L\left(u_{n}-\varphi_{n}, u_{n}-\varphi_{n}\right) \leq C\left\|\widetilde{u}_{n}\right\|_{p, \rho} \leq C \cdot L^{\frac{1}{2}}\left(u_{n}-\varphi_{n}, u_{n}-\varphi_{n}\right),
$$

and $u_{n}-\varphi_{n}$ is bounded in $H_{p, \rho}^{1}(\Omega, \Gamma)$, i.e., $\omega_{n}^{(1)}+\omega_{n}^{(2)}$ is bounded in $H_{p, \rho}^{1}(\Omega, \Gamma)$. Letting $\omega_{n}=\omega_{n}^{(1)}+\omega_{n}^{(2)}$, one has

$$
I^{\prime}\left(u_{n}\right) \omega_{n}=L\left(\omega_{n}, \omega_{n}\right)-\lambda_{k} \int_{\Omega} \omega_{n}^{2} \rho+\int_{\Omega} f\left(x, u_{n}\right) \omega_{n} \rho .
$$


By $(F-1)$, the Hölder inequality, and the Lebesgue dominated theorem, it is easy to show

$$
\int_{\Omega} f\left(x, u_{n}\right) \omega_{n} \rho \rightarrow 0, \quad \text { as } n \rightarrow \infty .
$$

Since $I^{\prime}\left(u_{n}\right) \rightarrow 0$ and $\omega_{n}$ is bounded in $H_{p, \rho}^{1}(\Omega, \Gamma)$, we have $I^{\prime}\left(u_{n}\right) \omega_{n} \rightarrow 0$ and

$$
L\left(\omega_{n}, \omega_{n}\right)-\lambda_{k} \int_{\Omega} \omega_{n}^{2} \rho \rightarrow 0, \quad \text { as } n \rightarrow \infty .
$$

Consequently, by virtue of Fatou's lemma, (4.1) and $(F-1)$, we have

$$
c \geq \int_{\Omega} \liminf _{n \rightarrow \infty} F\left(x, u_{n}\right) \rho=\int_{\Omega \cap\{\bar{u}>0\}} T^{+} \rho+\int_{\Omega \cap\{\bar{u}<0\}} T^{-} \rho,
$$

which contradicts with the condition $c<\inf _{\omega \in V\left(\lambda_{k}\right)}\left\{\int_{\Omega \cap\{\omega>0\}} T^{+} \rho+\int_{\Omega \cap\{\omega<0\}} T^{-} \rho\right\}$. Hence, the $(P S)_{c}$ sequence of the functional $I$ is bounded.

We can argue as the proof of Lemma 2.6, so the proof has been completed.

Lemma 4.2 Suppose that $(F-1)$ and $\left(F_{k}-2\right)$ are satisfied, $(\Omega, \Gamma)$ is a simple $V_{L}$-region and $\left(a_{i j}\right)=E$. Then the origin is a local minimum for the functional $I$.

Proof By $\left(F_{k}-2\right)$, we can choose $q \in\left(2, \frac{2 N}{N-1}\right)$ and a constant $C>0$ such that

$$
F(x, s) \geq \gamma s^{2}-C|s|^{q}, \quad \forall(x, s) \in \Omega \times \mathbb{R} .
$$

Consequently, by Remark 1.1 and Lemma 2.3, we have

$$
I(u) \geq \frac{1}{4}\left(1+\frac{2 \gamma}{\lambda_{1}}-\frac{\lambda_{k}}{\lambda_{1}}\right) c_{1}\|u\|_{p, \rho}^{2}
$$

$\|u\|_{p, \rho}<r$, where $r$ is small enough. Therefore the proof is completed.

Proof of Theorem 1.6 Since

$$
I\left(t \varphi_{1}\right) \leq\left(\lambda_{1}-\lambda_{k}\right) \frac{t^{2}}{2}+\int_{\Omega} k \rho \rightarrow-\infty
$$

as $t \rightarrow \infty$, we can choose $t^{+}>0$ and $t^{-}<0$, satisfying $\left\|t^{+} \varphi_{1}\right\|_{p, \rho}>r$ and $\left\|t^{-} \varphi_{1}\right\|_{p, \rho}>r$ such that $I\left(t^{+} \varphi_{1}\right)<0$ and $I\left(t^{-} \varphi_{1}\right)<0$, where $r$ is given by Lemma 4.2.

On the other hand, since $\varphi_{1}>0$, we know $t^{+} \varphi_{1}>0$ and $t^{-} \varphi_{1}<0$. Let $P_{+}=\left\{u \in H_{p, \rho}^{1}(\Omega, \Gamma) \mid\right.$ $u \geq 0\}$ and $P_{-}=\left\{u \in H_{p, \rho}^{1}(\Omega, \Gamma) \mid u \leq 0\right\}$. Consider the functionals $I^{ \pm}$, which are the restrictions of $I$ on $P_{ \pm}$. By Lemma 4.1 and $\left(F_{k}-1\right)$, for $c \geq 0$, we get $I^{ \pm}$satisfying $(P S)_{c}$ conditions. So by the mountain-pass lemma, we get two critical points $z^{ \pm} \in P_{ \pm}$. It is obvious that $z^{ \pm} \neq 0$. The proof has been completed. 
Authors' contributions

All authors collaborated and dedicated the same amount of time in working on this article. All authors read and approved the final manuscript.

\section{Acknowledgements}

The authors express their sincere thanks to the referees for their valuable criticisms of the manuscript and for helpful suggestions. This work was supported by National Natural Science Foundation of China (11171220) and Shanghai Leading Academic Discipline Project (XTKX2012)

Received: 18 January 2014 Accepted: 7 June 2014 Published online: 24 September 2014

\section{References}

1. Ahmad, S, Lazer, AC, Paul, JL: Elementary critical point theory and perturbations of elliptic boundary value problems at resonance. Indiana Univ. Math. J. 25, 933-944 (1976)

2. Bartsch, T, Chang, KC, Wang, ZQ: On the Morse indices of sign changing solutions of nonlinear elliptic problems. Math. Z. 233, 655-677 (2000)

3. Bartsch, T, Li, SJ: Critical point theory for asymptotically quadratic functionals and applications to problems with resonance. Nonlinear Anal. 28, 419-441 (1997)

4. Chang, KC: An extension of the Hess-Kato theorem to elliptic systems and its applications to multiple solutions problems. Acta Math. Sin. 15, 439-454 (1999)

5. Chang, KC: Infinite Dimensional Morse Theory and Multiple Solution Problems. Progress in Nonlinear Differential Equations and Their Applications. Birkhäuser, Boston (1993)

6. Chang, KC: Principal eigenvalue for weight in elliptic systems. Nonlinear Anal. 46, 419-433 (2001)

7. Costa, DG, Magalhes, CA: A variational approach to subquadratic perturbations of elliptic systems. J. Differ. Equ. 111, 103-121 (1994)

8. de Figueiredo, DG: Positive solutions of semilinear elliptic problems. In: Differential Equations (São Paulo, 1981) Lecture Notes in Math., vol. 957, pp. 34-87. Springer, Berlin (1982)

9. Furtado, MF, de Paiva, FOV: Multiplicity of solutions for resonant elliptic systems. J. Math. Anal. Appl. 319, 435-449 (2006)

10. Landesman, EM, Lazer, AC: Nonlinear perturbations of linear elliptic boundary value problems at resonance. J. Math Mech. 19, 609-623 (1970)

11. Pao, CV: Nonlinear Parabolic and Elliptic Equations. Plenum, New York (1992)

12. Shapiro, VL: Singular quasilinearity and higher eigenvalues. Mem. Am. Math. Soc. 153, 726 (2001)

13. Jia, G, Zhao, Q: Existence results in weighted Sobolev spaces for some singular quasilinear elliptic equations. Acta Appl. Math. 109, 599-603 (2010)

14. Jia, G, Sun, D: Existence of solutions for a class of singular quasilinear elliptic resonance problems. Nonlinear Anal. 74, 3055-3064 (2011)

15. Jia, G, Huang, LN, Zhang, XJ: Existence of solutions for quasilinear elliptic equations with superlinear nonlinearities. Bound. Value Probl. 2012, 90 (2012)

16. Willem, M: Minimax Theorems. Progress in Nonlinear Differential Equations and Their Applications. Birkhäuser, Boston (1996)

17. Rabinowitz, PH: On a class of nonlinear Schrödinger equations. Z. Angew. Math. Phys. 43, 270-291 (1992)

doi:10.1186/s13661-014-0156-9

Cite this article as: Jia and Zhang: Multiplicity of solutions for singular semilinear elliptic equations in weighted

Sobolev spaces. Boundary Value Problems 2014 2014:156.

\section{Submit your manuscript to a SpringerOpen ${ }^{\ominus}$ journal and benefit from:}

- Convenient online submission

- Rigorous peer review

Immediate publication on acceptance

- Open access: articles freely available online

- High visibility within the field

- Retaining the copyright to your article 